\title{
NGHIÊN CỨU GIẢI PHÁP KỸ THUẬT SỬ DỤNG CÔNG NGHẸ GNSS-RTK TRONG THI CÔNG XÂY DỬNG NHÀ SIÊU CAO TẦNG Ở VIẸTT NAM
}

\author{
TRẦN VIẾT TUẤN ${ }^{(1)}$, DIÊM CÔNG TRANG(2) \\ ${ }^{(1)}$ Truoòng đại học Mỏ - Địa chất Hà Nội \\ (2) Viện Khoa hoc Công nghệ Xây dưng
}

\section{Tóm tắt:}

Nội dung của bài báo trình bày kết quả nghiên cưu về một số giải pháp kỹ thuật cần ứng dụng khi sử dụng công nghệ GNSS-RTK để bố trí thi công xây dựng công trình nhà siêu cao tầng. Co sở lý thuyết, kết quả đo đạc thực nghiệm khi ứng dụng của các giải pháp kỹ thuật này nhằm nâng cao khả năng ưng dụng của công nghệ GNSS-RTK trong quá trình đảm bảo thi công các công trình xây dựng nhà cao tầng và siêu cao tầng ở Việt Nam.

\section{1. Đặt vấn đề}

Trong thi công xây dựng công trình nhà siêu cao tầng, để đảm bảo độ chính xác bố trí thi công xây dựng trong điều kiện công trình bị dao động do ảnh hưởng của các điều kiện ngoại cảnh và do bản thân tải trọng của công trình, chúng tôi đã đề nghị sử dụng công nghệ GNSS-RTK để xác định toạ độ tức thời của các điểm cơ sở trên các sàn thi công kết hợp với máy toàn đạc điện tử để bố trí chi tiết công trình [3]. Tuy nhiên khi sử dụng phương pháp bố trí này để bố trí chi tiết trên các sàn xây dựng cần phải xét đến yếu tố độ nghiêng của sàn thi công so với mặt phẳng nằm ngang do sự dao động của công trình gây ra [1] và cần phải tính chuyển kết quả đo GNSS-RTK tại thời điểm $\mathrm{t}_{\mathrm{i}}$ về hệ toạ độ thiết kế công trình nhằm đảm bảo yêu cầu: hệ toạ độ thi công xây dựng phải trùng với hệ toạ độ thiết kế công trình và sự biến dạng của chiều dài cạnh đo bằng công nghệ GNSSRTK có sự biến dạng nhỏ nhất so với chiều dài của cạnh trên mặt đất [2].

Chính vì vậy mà cần phải nghiên cứu bổ sung các giải pháp kỹ thuật cần thiết khi sử dụng công nghệ GNSS-RTK kết hợp với máy toàn đạc điện tử để bố trí thi công xây dựng các công trình nhà siêu cao tầng ở nước ta. \section{cứu}

2. Cơ sở lý thuyết và phương pháp nghiên

\subsection{Tính số hiệu chỉnh do độ nghiêng của sàn thi công vào số liệu bố trí công trình.}

- Giả sử tại thời điểm $\mathrm{t}_{1}$ vị trí toà nhà được xác định bởi các điểm $(\mathrm{A}, \mathrm{B}, \mathrm{C}, \mathrm{D})$ có toạ độ là $(\mathrm{x}, \mathrm{y})_{1}$.

- Tại thời điểm $t_{2}$ do ảnh hưởng của các yếu tố ngoại cảnh, các điểm $(\mathrm{A}, \mathrm{B}, \mathrm{C}, \mathrm{D})$ di chuyển đến vị trí $\left(\mathrm{A}_{1}, \mathrm{~B}_{1}, \mathrm{C}_{1}, \mathrm{D}_{1}\right)$ có toạ độ tức thời $(\mathrm{x}, \mathrm{y})_{2}$. Khi đó mặt sàn thi công bị nghiêng so với phương nằm ngang một góc $\gamma$ như (hình 1 ). Giá trị góc nghiêng $\gamma$ được xác định trực tiếp và liên tục bằng các cảm biến đo nghiêng gắn trực tiếp trên công trình [1].

- Nếu từ điểm M - điểm đặt máy toàn đạc điện tử tiến hành bố trí các điểm 1 và 2 theo toạ độ thiết kế dựa vào toạ độ tức thời của các điểm $\left(\mathrm{A}_{1}, \mathrm{~B}_{1}, \mathrm{C}_{1}, \mathrm{D}_{1}\right)$ thì cần phải tính số hiệu chỉnh vào khoảng cách bố trí do độ nghiêng $(\gamma)$ của mặt sàn gây ra

$$
\text { Theo công thức: } \quad \mathrm{S}_{\mathrm{bt}}=\frac{\mathrm{S}_{\mathrm{tk}}}{\cos \gamma}
$$

Trong đó: $\mathrm{S}_{\mathrm{tk}}$ là chiều dài cạnh thiết kế; $\mathrm{S}_{\mathrm{bt}}$ là chiều dài cạnh bố trí sau hiệu chỉnh

Như vậy, dựa vào kết quả đo góc nghiêng của sàn thi công $(\gamma)$ bằng các cảm biến đo nghiêng gắn trên công trình có thể tiến hành bố trí chi tiết

Ngày nhận bài: 05/10/2019, ngày chuyển phản biện: 09/10/2019, ngày chấp nhận phản biện: 15/10/2019, ngày chấp nhận đăng: 18/10/2019 
theo các khoảng cách nghiêng đã hiệu chỉnh $\left(\mathrm{S}_{\mathrm{bt}}\right)$ hoặc đo theo chế độ đo khoảng cách nằm ngang có sẵn trên máy toàn đạc khi bố trí công trình $\left(\mathrm{S}_{\mathrm{tk}}\right)$.

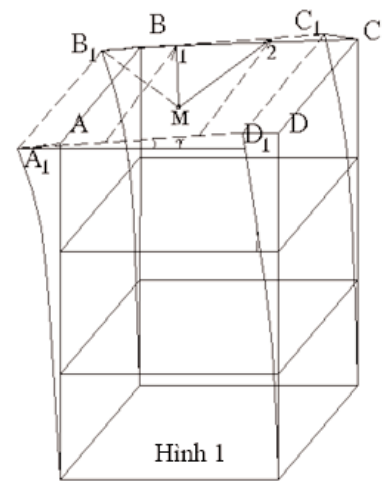

Vấn đề cần đặt ra ở đây là xem giới hạn của góc nghiêng $(\gamma)$ để cần phải tính số hiệu chỉnh khoảng cách. Từ (1) ta có: $\mathrm{S}_{\mathrm{tk}}=\mathrm{S}_{\mathrm{bt}} \cos \gamma$

Tiến hành biến đổi:

$$
\begin{aligned}
& \mathrm{S}_{\mathrm{tk}}-\mathrm{S}_{\mathrm{bt}}=\mathrm{S}_{\mathrm{bt}} \cos \gamma-\mathrm{S}_{\mathrm{bt}} \\
& \Delta_{\mathrm{S}}=\mathrm{S}_{\mathrm{bt}}(\cos \gamma-1) \\
& \Delta_{\mathrm{S}}=\mathrm{S}_{\mathrm{bt}} 2 \sin ^{2} \frac{\gamma}{2} \text { Suy ra: } \quad \gamma=\rho^{\prime \prime} \sqrt{\frac{\Delta_{\mathrm{s}}}{\mathrm{S}}}
\end{aligned}
$$

Tính giá trị $\Delta_{\mathrm{S}}$ cho phép

Từ hình 1: Sai số tổng hợp của điểm 1 được tính như sau:

$$
\mathrm{m}_{1}^{2}=\mathrm{m}_{\mathrm{M}}^{2}+\mathrm{m}_{\mathrm{bt}}^{2}+\mathrm{m}_{\gamma}^{2}
$$

Trong đó: $\mathrm{m}_{\mathrm{bt}}$ : là sai số bố trí điểm 1 bằng máy toàn đạc điện tử;

$\mathrm{m}_{\gamma}$ : sai số bố trí điểm 1 do góc nghiêng của sàn $(\gamma)$ tạo ra;

$\mathrm{m}_{\mathrm{M}}$ : sai số của điểm đặt máy $\mathrm{M}$

Để có thể bỏ qua các giá trị $\mathrm{m}_{\gamma}$ và $\mathrm{m}_{\mathrm{M}}$ thì yêu cầu ảnh hưởng của các nguồn sai số này không vượt quá $10 \%$ của sai số tổng hợp tức là:

$$
\mathrm{m}_{\mathrm{M}}=\mathrm{m}_{\gamma}=\frac{\mathrm{m}_{\mathrm{bt}}}{\mathrm{k}} \quad(\mathrm{k}=2)
$$

Thay vào công thức (3) và biến đổi ta có

$$
\mathrm{m}_{\mathrm{bt}}=\frac{\mathrm{m}_{\mathrm{l}} \mathrm{k}}{\sqrt{2+\mathrm{k}^{2}}}
$$

Nếu lấy sai số trung phương tương hỗ giữa hai điểm cần bố trí 1 và 2 là $\left(\mathrm{m}_{\mathrm{th}}\right)_{1-2} \leq \pm 3 \mathrm{~mm}$ [3],

Ta có $\mathrm{m}_{1}= \pm 2.1 \mathrm{~mm}$; theo $(5): \mathrm{m}_{\mathrm{bt}}= \pm 1.7$ $\mathrm{mm}$; và $\mathrm{m}_{\gamma}= \pm 0.9 \mathrm{~mm}$

Sử dụng (2) để tính ta thấy: Khi khoảng cách bố trí $\mathrm{S} \leq 100 \mathrm{~m}$ thì $\gamma \leq 14$ '

Nhận xét: khi bố trí chi tiết trên các sàn thi công tại các công trình nhà siêu cao tầng, nếu góc nghiêng $\gamma$ của sàn thi công không vượt quá 14 ' thì không cần phải tính số hiệu chỉnh này vào chiều dài cạnh bố trí chi tiết công trình.

2.2. Tính chuyển kết quả đo GNSS-RTK về hệ tọa độ thiết kế của công trình

Tại thời điểm $\mathrm{t}_{\mathrm{i}}$ bằng kết quả đo GNSS-RTK cho ta toạ độ tại các điểm $\left(\mathrm{A}_{1}, \mathrm{~B}_{1}, \mathrm{C}_{1}, \mathrm{D}_{1}\right)$ ở dạng toạ độ địa tâm $(\mathrm{X}, \mathrm{Y}, \mathrm{Z})$ i và toạ độ trắc địa $(\mathrm{B}, \mathrm{L}$, $\mathrm{H})_{\mathrm{i}}$ hoặc toạ độ $(\mathrm{x}, \mathrm{y}, \mathrm{h})_{\mathrm{i}}$ trong hệ toạ độ VN 2000. Trong khi đó công trình lại được xây dựng trên mặt đất và sử dụng hệ toạ độ đã dùng để thiết kế công trình công trình (thường là hệ toạ độ phẳng, giả định). Chính vì vậy để sử dụng các kết quả đo GNSS-RTK ở thời điểm $\mathrm{t}_{\mathrm{i}}$ để bố trí các điểm chi tiết điểm 1 và 2 (hình 1 ) trên công trình cần phải thực hiện phép tính chuyển toạ độ kết quả đo GNSS-RTK về hệ toạ độ thiết kế và thi công của công trình.

Có nhiều phương pháp tính chuyển tọa độ GNSS ở đây chúng tôi chọn phương pháp tính chuyển toạ độ GNSS về hệ toạ độ thi công công trình qua hệ toạ độ địa diện chân trời có nguyên lý như sau [2]:

a. Từ kết quả đo GNSS-RTK cho ta tọa độ trong hệ toạ độ địa tâm của điểm $\mathrm{j}(\mathrm{X}, \mathrm{Y}, \mathrm{Z})_{\mathrm{j}}$ tiến hành tính chuyển toạ độ địa tâm của điểm $\mathrm{j}$ về hệ toạ độ địa diện chân trời theo công thức [2]:

$\left[\begin{array}{l}\mathrm{x} \\ \mathrm{y} \\ \mathrm{z}\end{array}\right]=\left[\begin{array}{ccc}-\sin \mathrm{B}_{0} \cdot \cos \mathrm{L}_{0} & -\sin \mathrm{B}_{0} \cdot \sin \mathrm{L}_{0} & \cos \mathrm{B}_{0} \\ -\sin \mathrm{L}_{0} & \cos \mathrm{L}_{0} & 0 \\ \cos \mathrm{B}_{0} \cdot \cos \mathrm{L}_{0} & \cos \mathrm{B}_{0} \cdot \sin \mathrm{L}_{0} & \sin \mathrm{B}_{0}\end{array}\right] \cdot\left[\begin{array}{c}\mathrm{X}_{\mathrm{j}}-\left(\mathrm{N}_{0}+\mathrm{H}_{0}\right) \cdot \cos \mathrm{B}_{0} \cdot \cos \mathrm{L}_{0} \\ \mathrm{Y}_{\mathrm{j}}-\left(\mathrm{N}_{0}+\mathrm{H}_{0}\right) \cdot \cos \mathrm{B}_{0} \cdot \sin \mathrm{L}_{0} \\ \mathrm{Z}_{\mathrm{j}}-\left[\mathrm{N}_{0}\left(1-\mathrm{e}^{2}\right)+\mathrm{H}_{0}\right] \cdot \sin \mathrm{B}_{0}\end{array}\right]$

Trong đó: - $\left(\mathrm{X}_{\mathrm{j}}, \mathrm{Y}_{\mathrm{j}}, \mathrm{Z}_{\mathrm{j}}\right)$ là tọa độ vuông góc 
không gian địa tâm của điểm GNSS cần tính chuyển

- $\left(\mathrm{B}_{0}, \mathrm{~L}_{0}, \mathrm{H}_{0}\right)$ là điểm gốc của hệ toạ độ địa diện chân trời.

- $\mathrm{N}_{0}$ là bán kính cong vòng thẳng đứng thứ nhất đi qua điểm gốc của hệ toạ độ địa tâm [2]

Khi áp dụng cho công trình nhà siêu cao tầng, nên chọn gốc hệ toạ độ địa diện chân trời như sau

$$
\mathrm{B}_{0}=\frac{\sum \mathrm{B}_{\mathrm{j}}}{\mathrm{n}}
$$

Với $\mathrm{n}$ là số điểm đo GNSS trong lưới bố trí.

Giá trị độ cao của gốc hệ toạ độ địa diện $\left(\mathrm{H}_{0}\right)$ chọn bằng độ cao bề mặt móng công trình.

b. Sử dụng phép tính chuyển Helmert để tính chuyển toạ độ các điểm đo GNSS tại thời điểm $t$ từ hệ toạ độ địa diện chân trời về hệ toạ độ thiết kế và thi công của công trình dựa vào các điểm song trùng [2].

\section{Kết quả đo đạc và tính toán thực nghiệm}

Để minh hoạ cho những nội dung nghiên cứu về lý thuyết đã nêu trên, chúng tôi đã tiến hành đo đạc tại khu vực Viện Khoa học Công nghệ Xây dựng với hệ thống máy thu của hãng Trimble: GNSS R8S

- Thành lập trên mặt đất một lưới đo góc-cạnh bao gồm 8 điểm như (hình 2);

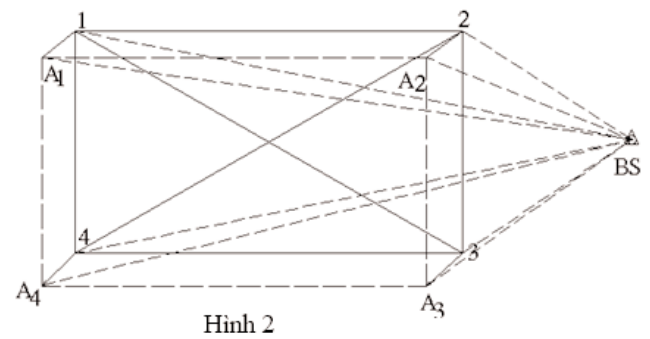

- Dùng máy toàn đạc điện tử TCR-1201 (độ chính xác đo góc cạnh là: $\mathrm{m}_{\beta}= \pm 1$ "; $\mathrm{m}_{\mathrm{S}}= \pm 1,5$ $\mathrm{mm}$ ) và thước thép đo góc và cạnh trong lưới; xử lý số liệu trong hệ toạ độ giả định được coi là hệ toạ độ thiết kế và thi công của công trình.

Dùng 05 máy thu GNSS R8S (Trimble): 01 máy đặt tại điểm BS là điểm trạm base đã có toạ độ và độ cao trong hệ toạ độ VN-2000; 04 máy thu GNSS 8S đặt tại các điểm $(1,2,3,4)$, (A1, A2, A3, A4) đo theo chế độ đo tĩnh (hai ca đo). Kết quả đo GNSS tĩnh cho như cột (1), (2) trên bảng 1 .

Sau đó chuyển sang chế độ đo GNSS-RTK với trạm base đặt tại điểm BS với tần suất thu tín hiệu là 1 " và thời gian thu tại các điểm đo khoảng 10'. Sau khi xử lý số liệu có kết quả đo GNSS-RTK như phần 2 các cột (1), (2) trong bảng 1 .

Lấy toạ độ đo GNSS tĩnh tại các điểm 3 và 4 làm toạ độ khởi tính để tính toán số liệu lưới đo góc cạnh trên mặt đất theo hệ toạ độ VN2000. Kết quả đo cho như cột (3), (4) của bảng 1 . Toạ độ các điểm trong lưới lấy theo hệ toạ độ thiết kế công trình cho như các cột (1), (2) của bảng 2 .

- Độ lệch toạ độ của các điểm trong lưới đo bằng các phương pháp đo khác nhau thể hiện trên cột (5). (6), (7) trên bảng 1.

Nhận xét: Từ kết quả so sánh toạ độ các điểm của lưới thực nghiệm đo theo các phương pháp khác nhau trình bày trên bảng 1 và giá trị toạ độ thiết kế các điểm của lưới trên bảng 2 ta thấy: (Xem bảng 1)

Không thể sử dụng trực tiếp toạ độ các điểm đo GNSS-RTK trên các sàn thi công để xác định toạ độ điểm đặt máy toàn đạc điện tử và bố chí chi tiết công trình được vì các lý do sau đây:

- Sự khác biệt về hệ toạ độ giữa hệ toạ độ đo bằng GNSS-RTK (hệ VN 2000) và hệ toạ độ thiết kế và thi công công trình (hệ giả định).

- Sự biến dạng chiều dài các cạnh của lưới đo bằng GNSS-RTK so với chiều dài cạnh trên mặt đất là tương đối lớn, vượt quá giới hạn độ chính xác cho phép bố trí chi tiết công trình - Trên bảng 1: Độ lệch toạ độ từ $3 \mathrm{~mm} \div 9 \mathrm{~mm}$.

Chính vì vậy mà cần phải tiến hành tính chuyển toạ độ từ hệ toạ độ địa tâm khi đo GNSSRTK về hệ toạ độ thiết kế và thi công công trình. (Xem bảng 2) 
Ở đây chúng tôi đã sử dụng phương pháp tính chuyển kết quả đo GNSS về hệ toạ độ thi công theo phương pháp tính chuyển qua hệ toạ độ địa diện chân trời [2] bằng cách sử dụng thuật toán tính chuyển (6), (7) kết hợp với phép tính chuyển Helmert. Kết quả tính chuyển được trình bày trong các (3), (4) của bảng 2 . Từ kết quả so sánh trong bảng 2 cho thấy: khi thực phép tính chuyển toạ độ đo GNSS-RTK từ hệ toạ độ địa tâm về hệ toạ độ thi công trình qua hệ tọa độ địa diện chân trời sẽ đáp ứng được các yêu cầu:

- Các điểm đo GNSS-RTK dùng làm điểm gốc giao hội trên các sàn thi công sẽ có tọa độ trùng với hệ toạ độ đã dùng để thiết kế và thi công công trình.

- Sự biến dạng về chiều dài cạnh lưới (hoặc sai số xác định toạ của các điểm đo GNSS-RTK) có giá trị nhỏ nằm trong giới hạn cho phép về độ

Bảng 1: Kết quả đo xác định toạ độ luới thực nghiệm theo các phưong pháp đo khác nhau

\begin{tabular}{|c|c|c|c|c|c|c|c|}
\hline \multirow{3}{*}{ TT } & \multicolumn{2}{|c|}{ GPS TĨNH } & \multicolumn{2}{|c|}{ LƯỚI ĐO GÓC-CẠH } & \multirow{2}{*}{$\underset{(\mathrm{m})}{\Delta \mathrm{X}}$} & \multirow{2}{*}{$\begin{array}{l}\Delta \mathrm{Y} \\
(\mathrm{m})\end{array}$} & \multirow{2}{*}{$\begin{array}{l}\Delta \mathrm{S} \\
(\mathrm{m})\end{array}$} \\
\hline & $\mathrm{X}(\mathrm{m})$ & $\mathrm{Y}(\mathrm{m})$ & $\mathrm{X}(\mathrm{m})$ & $\mathrm{Y}(\mathrm{m})$ & & & \\
\hline & (1) & (2) & (3) & (4) & (5) & (6) & (7) \\
\hline $\mathrm{A} 1$ & 2328533.453 & 581781.627 & 2328533.454 & 581781.630 & -0.001 & -0.003 & 0.0032 \\
\hline $\mathrm{A} 2$ & 2328530.272 & 581716.662 & 2328530.280 & 581716.659 & -0.008 & 0.003 & 0.0085 \\
\hline $\mathrm{A} 3$ & 2328554.318 & 581716.58 & 2328554.318 & 581716.580 & 0 & 0 & 0.0000 \\
\hline $\mathrm{A} 4$ & 2328553.32 & 581777.104 & 2328553.317 & 581777.104 & 0.003 & 0 & 0.0030 \\
\hline 1 & 2328533.591 & 581781.789 & 2328533.592 & 581781.793 & -0.001 & -0.004 & 0.0041 \\
\hline \multirow[t]{2}{*}{2} & 2328530.448 & 581716.783 & 2328530.451 & 581716.790 & -0.003 & -0.007 & 0.0076 \\
\hline & \multicolumn{2}{|c|}{ GNSS-RTK } & & & & & \\
\hline $\mathrm{A} 1$ & 2328533.453 & 581781.627 & 2328533.454 & 581781.630 & -0.001 & -0.003 & 0.0030 \\
\hline $\mathrm{A} 2$ & 2328530.272 & 581716.662 & 2328530.28 & 581716.659 & -0.008 & 0.003 & 0.0085 \\
\hline $\mathrm{A} 3$ & 2328554.318 & 581716.580 & 2328554.318 & 581716.580 & 0.000 & 0.000 & 0.0002 \\
\hline 1 & 2328533.593 & 581781.788 & 2328533.592 & 581781.793 & 0.001 & -0.005 & 0.0051 \\
\hline 2 & 2328530.447 & 581716.782 & 2328530.451 & 581716.790 & -0.004 & -0.008 & 0.0089 \\
\hline
\end{tabular}

Bảng 2: So sánh toạ độ các điểm lưới thực nghiệm sau tính chuyển

\begin{tabular}{|c|c|c|c|c|c|c|c|}
\hline \multirow{3}{*}{ TT } & \multicolumn{2}{|c|}{ Toạ độ thiết kế } & \multicolumn{2}{|c|}{$\begin{array}{l}\text { Toạ độ đo GNSS-RTK sau } \\
\text { tính chuyê̂n }\end{array}$} & \multirow{2}{*}{$\begin{array}{r}\Delta \mathrm{X} \\
(\mathrm{m})\end{array}$} & \multirow{2}{*}{$\begin{array}{l}\Delta \mathrm{Y} \\
(\mathrm{m})\end{array}$} & \multirow{2}{*}{$\begin{array}{l}\Delta \mathrm{S} \\
(\mathrm{m})\end{array}$} \\
\hline & $\mathrm{X}(\mathrm{m})$ & $\mathrm{Y}(\mathrm{m})$ & $\mathrm{X}(\mathrm{m})$ & $\mathrm{Y}(\mathrm{m})$ & & & \\
\hline & (1) & (2) & (3) & (4) & $(5)$ & (6) & $(7)$ \\
\hline $\mathrm{A}_{1}$ & 995.294 & 480.061 & 995.297 & 480.062 & 0.003 & 0.001 & 0.003 \\
\hline $\mathrm{A}_{2}$ & 1060.201 & 475.824 & 1060.199 & 475.821 & -0.001 & -0.003 & 0.003 \\
\hline $\mathrm{A}_{3}$ & 1060.675 & 499.857 & 1060.675 & 499.857 & 0 & 0 & 0 \\
\hline $\mathrm{A}_{4}$ & 1000.145 & 499.849 & 1000.145 & 499.851 & 0 & 0.002 & 0.002 \\
\hline 1 & 995.133 & 480.202 & 995.136 & 480.203 & 0.003 & 0.001 & 0.003 \\
\hline 2 & 1060.073 & 475.997 & 1060.075 & 475.995 & 0.002 & -0.002 & 0.003 \\
\hline 3 & 1060.513 & 500.000 & 1060.513 & 500.000 & $\begin{array}{c}\text { Điểm song } \\
\text { trùng }\end{array}$ & & \\
\hline 4 & 1000.000 & 500.000 & 1000.000 & 500.000 & $\begin{array}{c}\text { Điềm song } \\
\text { trùng }\end{array}$ & & \\
\hline
\end{tabular}


xác định tọa độ của các điểm đo GNSS-RTK dùng làm cơ sở để bố trí chi tiết trên các sàn thi công nhà siêu cao tầng ở nước ta. $\bigcirc$

\section{Tài liệu tham khảo}

[1]. Ngô Văn Hiếu và nnk (2018), "Sử dụng hệ thống VAS trong việc đảm bảo độ thẳng đứng của toà nhà siêu cao tầng LAND MARK 81 TP Hồ Chí Minh", Tuyển tập báo cáo hội nghị khoa học quốc tế ACI, Viện khoa hoc công nghệ xây dưng, Hà Nội
[2]. Trần Viết Tuấn (2005), "Nghiên cứu phương pháp tính chuyển tọa độ các điểm đo GPS về hệ tọa độ thi công công trình", Tạp chí KHKT Mỏ - Địa chất số 11/7-2005, Hà Nội.

[3]. Trần Viết Tuấn, Diêm Công Trang (2019), "Nghiên cứu ứng dụng công nghệ GNSS-RTK trong thi công xây dựng nhà siêu cao tầng ở Việt Nam“, Tạp chi Khoa học Đo đạc và Bản đồ, số 40 tháng 6/2019, Hà Nội.O

\section{Summary}

\section{Research on technical solution of GNSS-RTK technology in construction of super high-rise building in Vietnam}

Tran Viet Tuan, Hanoi University of Mining and Geology

Diem Cong Trang, Vietnam Institute for Building Science and Technology

The content of the article presents the research results of some technical solutions to be applied when using GNSS-RTK technology to arrange the construction of super-high-rise buildings. Theoretical basis, experimental measurement results during application of these technical solutions to improve the applicability of GNSS-RTK technology in the process of ensuring construction of high-rise buildings and super high-rise in Vietnam. $O$

\section{ĐO SÂU ĐỊA HÌNH ĐÁY BIỂN VÙNG NƯỚC NÔNG.......}

\section{(Tiếp theo trang 38)}

[5]. Dietrich 2017, Bathymetric Structurefrom-Motion: Extracting shallow stream bathymetry from multi-view stereo photogrammetry, Earth Surfaces Processes and Landforms,

Vol. 45, pp. 355 - 364.

[6]. Seeber, G. 2003, Satellite Geodesy, $2^{\text {nd }}$ edition, Walter de Gruyter, Berlin, 2003.O

\section{Summary}

Shallow-water Bathymetry by stereo photogrammetric approach using satellite imageries WorldView-2, experimental case in submerged reef Hai Sam, Spratly Islands, Vietnam

Nguyen Ha Phu, National Remote Sensing Department

Pham Minh Hai, Institute of Geodesy and Cartography

Nguyen Trong Truong Son, Hanoi University of Natural Resources and Environment

Satellite multispectral image data is being applied effectively in bathymetry in shallow water areas. Most of studies are focused on determining the water depth using the radiometric approach. However, with the acquiring ability of stereo image data in a single pass from WorldView-2 satellite, the photogrammetric approach is becoming more interestingly because the advantage of without using In-Situ data. In this experimental case, the near-shore bathymetry by stereo photogrammetryusing WorldView-2 satellite data is conducted at the coral reef Hai Sam that belong to Spratly Islands (Vietnam). After removing the affects of atmosphere and sun-glint as well as adjusting the depth difference caused by refraction at the water/air interface, the achieved depth can be up to over $42,0 \mathrm{~m}$. These results show that the applicability of shallow-water bathymetry by stereo photogrammetry can be applied spreadly to other islands, islets and surmerged reefs at the Paracel Islands and the Spratly Islands. $\bigcirc$ 